\title{
Characterization and Optimization of Semiconductor Specimen Preparation for QHREM
}

\author{
N.Y. Jin-Phillipp, M. Kelsch, F. Phillipp, and M. Rühle \\ Max-Planck-Institut für Metallforschung, Heisenbergstr.3, D-70569 Stuttgart, Germany
}

The quality of TEM specimen is one of the crucial factors of successful electron microscopy, especially in the case of quantitative high-resolution electron microscopy (QHREM) on an atomic scale. The amorphous over-layers, the thickness of which is related to the conditions of preparation, limit the accuracy of the determination of the image maximum positions. In the present work the conditions of low-energy ion (LE) polishing on the basis of optimized specimen preparation of III$\mathrm{V}$ semiconductors has been studied. The quality of the LE-polished specimens has been evaluated by QHREM using an image processing package LADIA (LAttice DIstortion Analysis).[1-3]

GaAs $\{110\}$ cross-sectional specimens are prepared with an optimized process. This involves not only suitable parameters for ion-milling (3kV 10-12 $\mu$ A before perforation) and ion-polishing (1.7kV 40min) with GATAN PIPS 691, but also precise knowledge of the specimen thickness before ion-milling, as well as perfect specimen surface finishing by multi-step grinding, dimpling, and polishing. [Higher gun-voltage, e.g. $3.5 \mathrm{kV}$ in the case of specimen $\mathrm{D}$, without any ion-polishing leads to thick amorphous over-layers and defect formation, as pointed by an arrow in Fig.1(a), due to ion damage.] After HREM imaging, the specimens are further LE-polished with various ion energies and time duration (Table 1) using Technoorg Linda.

Fig.1 compares the HREM image taken after LE-polishing [Fig.1(b)] with that before LE-polishing [Fig.1(a)] of the same specimen D. Defects and thick amorphous layers are succ essfully removed, and the image quality is higher. Fig. 2 demonstrates HREM images of specimen $\mathrm{C}$ before and after LE-polishing of medium voltage and duration. Specimen quality is evaluated by QHREM. The distances between intensity maximum positions along [001] direction, $d$, are determined with a sampling rate of $70 \mathrm{pixel} / \mathrm{nm}$ [Fig.3(a)]. The width of the distribution of $d(2 \sigma)$ is used to estimate the accuracy of the measurements.[4] Fig.3(b) shows the histogram of $d$ measured from Fig.2. The distribution obtained after LE-polishing is much narrower, indicating an improvement of the accuracy of the measurements. In the Table 1, the results from specimens LE-polished at various conditions are summarized and compared with those obtained before LE-polishing. The quality of specimens is markedly improved by choosing suitable parameters of LE-polishing. The standard deviation $\sigma$ is reduced by a factor two and three in specimen $\mathrm{C}$ and $\mathrm{D}$ respectively. It should also be noted that for well ion-milled specimens (e.g., sample A) further LE-polishing might lead to degradation, and is therefore not necessary.

In summary, the preparation of cross-sectional TEM specimens of III-V semiconductors is optimized to meet the need of HREM. Further LE-polishing with proper parameters improves the results of QHREM up to a factor of two, when routine ion-milling alone is not satisfactory.

\section{References}

[1] N.Y. Jin-Phillipp and F. Phillipp, J. Microscopy 194 (1999) 161

[2] N.Y. Jin-Phillipp and F. Phillipp, J. Appl. Phys. 88 (2000) 710

[3] K. Du et al, J. Mat. Sci. Technol. 18 (2002), in press

[4] H. Seitz, et al, J. Microscopy 190 (1998) 184 
Table $1 \quad$ Parameters and summary of QHREM results of LE-polishing

\begin{tabular}{|c|c|c|c|c|c|}
\hline Specimen & Voltage $(\mathrm{kV})$ & Duration $(\min )$ & $\sigma_{0}(\mathrm{~nm})$ & $\sigma_{e}(\mathrm{~nm})$ & Result \\
\hline A & 0.25 & 20 & - & - & more amorphous \\
\hline B & 0.25 & 40 & 0.0240 & 0.0188 & fair \\
\hline C & 0.35 & 40 & 0.0207 & 0.0112 & good \\
\hline D & 0.5 & 30 & $0.0407^{+}$ & 0.0125 & good \\
\hline$E^{*}$ & 0.5 & 60 & 0.0229 & 0.0222 & fair \\
\hline
\end{tabular}

${ }^{+}$Thick amorphous layers and defects due to ion-damage during ion-milling with $3.5 \mathrm{kV}$ in PIPS.

* Specimen E was polished with a reconstructed Baltec RES 010

$\sigma_{0}$ : standard deviation before LE-polishing, $\sigma_{e}$ : standard deviation after LE-polishing.
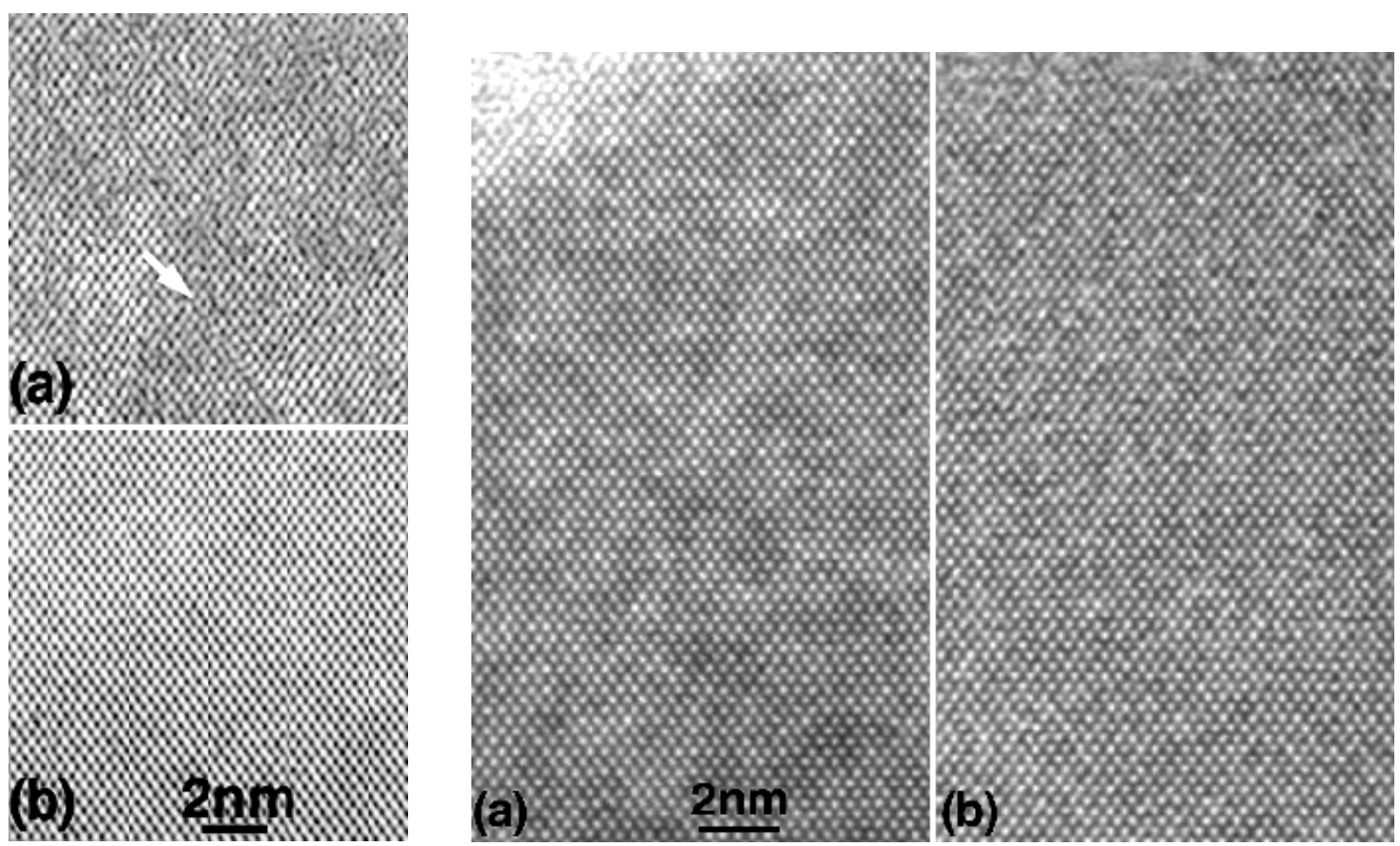

Fig1. HREM from specimen D. Fig.2 Images of specimen C before (a) and after (b) LE-polishing for QHREM.
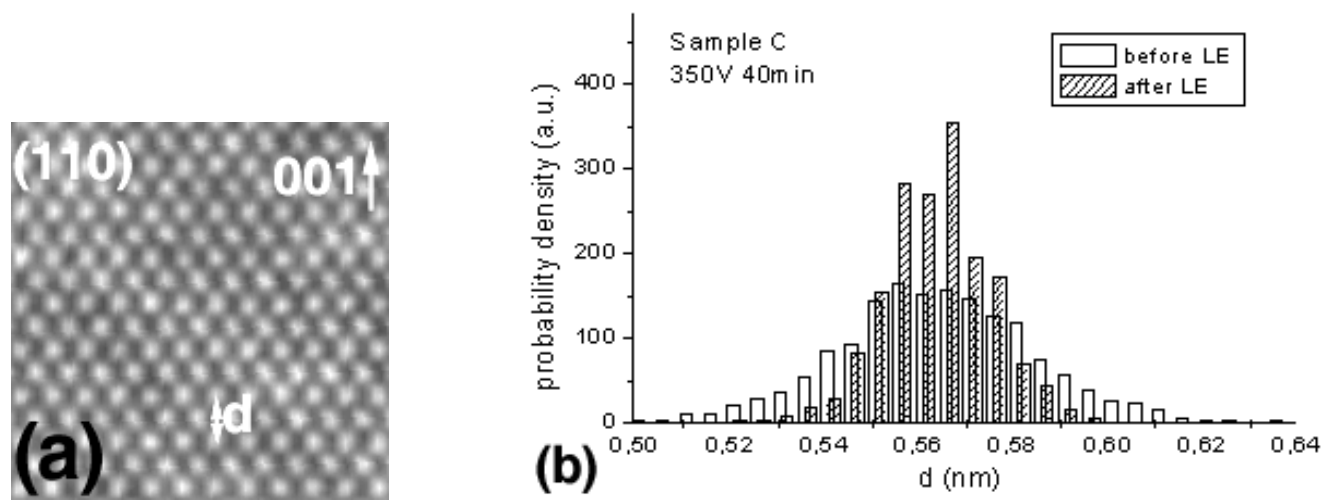

Fig.3 (a) Schematic of QHREM, (b) Distribution of $d$ measured from specimen C. 